\title{
Can commercial ferrofluids be exploited in AC magnetic hyperthermia treatment to address diverse biomedical aspects?
}

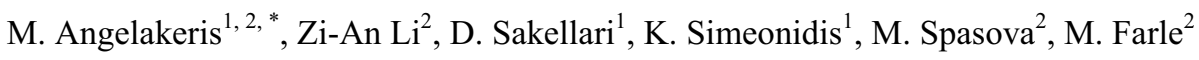 \\ ${ }^{1}$ Department of Physics, Aristotle University of Thessaloniki, 54 124, Greece \\ ${ }^{2}$ Fakultät für Physik and Center for Nanointegration Duisburg-Essen (CeNIDE), Universität Duisburg-Essen, Lotharstr. 1, \\ Duisburg D-47048, Germany
}

\begin{abstract}
Multifunctional magnetic nanoparticles are considered as promising candidates for various applications combining diagnosis, imaging and therapy. In the present work, we elaborate on the commercial colloidal solution "FluidMAG" (from Chemicell $\mathrm{GmbH}$ ) as a possible candidate for magnetic hyperthermia application. The current product is a dispersion of magnetite nanoparticles employed for purification or separation of biotinylated biomolecules from different sources (e.g. blood). Transmission Electron Microscopy showed that the NPs have a spherical shape with mean diameter of $12.3 \mathrm{~nm}( \pm 20 \%)$, and SQUID magnetometry revealed their superparamagnetic character. Our promising results of the AC hyperthermia efficiency of "FluidMAG" suggest that with the appropriate manipulation it can also be exploited as magnetic hyperthermia agent.
\end{abstract}

\section{Introduction}

Many research groups worldwide are exploring the potential of magnetic nanoparticles (MNPs) as biomedical magnetism-driven carriers in biomedical applications [1]. Most of these studies utilize MNPs synthesized in-house, aiming in the facile sample control and property tuning, while issues like formulation, reproducibility, toxicity and quality assurance are usually postponed for the later stages when MNPs will actually be introduced to clinical trials. AC hyperthermia is one of the diverse modalities under study, which is a cancer therapy, involving the targeted administration of MNPs into the body, their accumulation at sites of cancer, and their local heating with an externally applied AC magnetic field. AC magnetic hyperthermia continues to gain interest as a synergistic approach of a least invasive character together with facile remote control of activation and multifunctional potential in diverse magnetismdriven treatments.[1] Thus, it follows the current theranostics trend that requires multifunctional MNPs, possessing more than one magnetically triggered modality.

Contrary to home-made MNPs, commercially produced materials, particularly those certified to Good Manufacturing Process (GMP) standards may be rapidly pushed through the medical approval processes since commercial drive and company resources are focusing in the delivery of the new therapy to the patient as rapidly as possible. Additionally, the biocompatibility and toxicity aspects of such products have usually been resolved prior to their commercial exploitation.

Following a phenomenologically contradictious survey, Kallumadil et al [2] examined a series of commercially available biomedical MNPs as possible candidates for hyperthermia applications. The major advantage of such a study is that an optimum system directly addresses the multifunctional role in modern theranostics and may be further implemented in therapies with faster steps since major tasks have already been undertaken.

Table 1. Particle and Functionality parameters of fluidMAGStreptavidin.

\begin{tabular}{|c|c|c|}
\hline \multicolumn{3}{|c|}{ Particle } \\
\hline Hydrodynamic diameter: & $100 \mathrm{~nm}$ & $200 \mathrm{~nm}$ \\
\hline Number of Particles: & $\sim 1.8 \times 10^{15} / \mathrm{g}$ & $\sim 2.2 \times 10^{14} / \mathrm{g}$ \\
\hline Density: & $\sim 1.25 \mathrm{~g} / \mathrm{cm}^{3}$ & \\
\hline Matrix: & Starch & \\
\hline Magnetic Type: & Superparama & etic $(40 \mathrm{emu} / \mathrm{g})$ \\
\hline \multicolumn{3}{|c|}{ Functionality } \\
\hline Functional Group: & \multicolumn{2}{|l|}{ Streptavidin } \\
\hline Binding Canacity & \multicolumn{2}{|c|}{80 pmol biotinylated protein } \\
\hline $\begin{array}{r}\text { per } 1 \text { mg fluidMAG- } \\
\text { Streptavidin: }\end{array}$ & \multicolumn{2}{|c|}{$\begin{array}{l}150 \text { pmol biotinylated } \\
\text { oligonucleotide }\end{array}$} \\
\hline Storage Buffer & \multicolumn{2}{|c|}{$\begin{array}{l}\text { PBS, } 0.1 \% \text { BSA, } 0.05 \% \text { sodium } \\
\text { azide }\end{array}$} \\
\hline
\end{tabular}


In this work, we examined a commercial colloidal solution based on $\mathrm{Fe}_{3} \mathrm{O}_{4}$, a widely studied and currently used biomedical agent also in clinical practice $[3,4]$. The sample under study is the commercial ferrofluid named fluidMAG-Streptavidin (purchased from the company: Chemicell GmbH, Eresburgstrasse 22-23, 12103 Berlin, Germany). [5] According to its accompanying datasheet, the sample is a $5 \mathrm{ml}-10 \mathrm{mg} / \mathrm{mL}$ aqueous dispersion of magnetite nanoparticles to be employed for purification or separation of biotinylated biomolecules, including antigens, antibodies or nucleic acids from different sources such blood, sera, tissues and food. Additional parameters from its datasheet are summarized in Table 1 separated in particle and functionality parameters.

\section{Experimental}

The structural and morphological properties of the particles were investigated ex-situ by high resolution and scanning transmission electron microscopy (HRTEM and STEM). We used a Philips Tecnai F20 Supertwin microscope operated at $200 \mathrm{kV}$ with a field emission gun and a double Cs-corrected JEOL JEM-2200FS microscope.
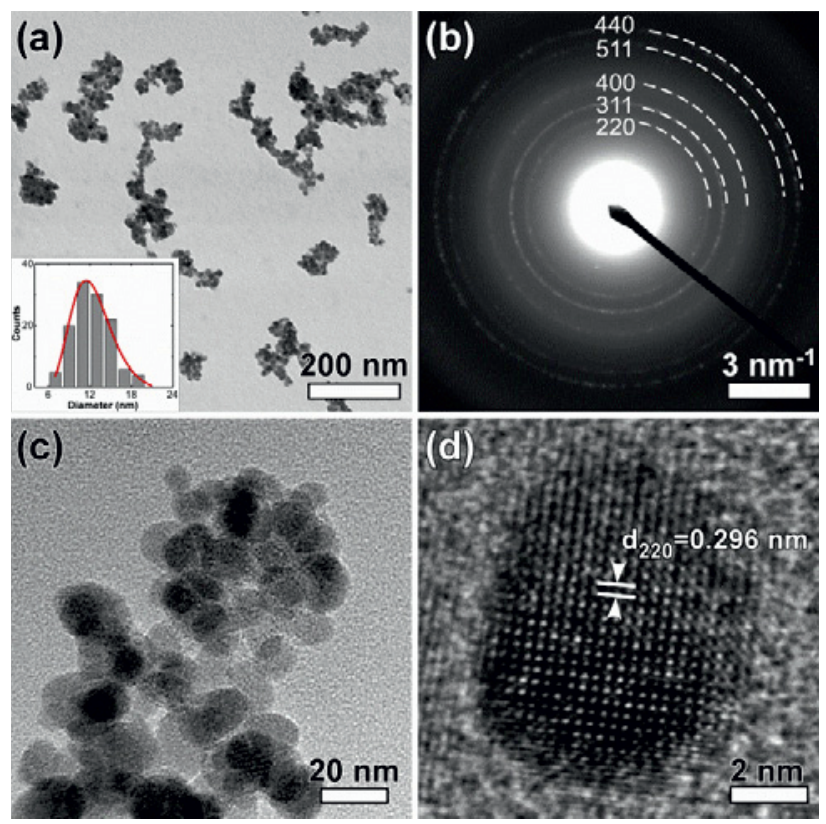

Fig. 1. TEM imaging and corresponding analysis of fluidMAGStreptavidin. (a) Conventional TEM image showing agglomerates of varying size $(100-200 \mathrm{~nm})$ and the log-normal size distribution (inset) of constituent nanoparticles of average diameter $12.3 \mathrm{~nm} \pm 20 \%$. (b) Electron Diffraction Pattern identifying magnetite phase. (c) HRTEM image of agglomerates showing the overlapping nanoparticles. (d) HRTEM image reconstruction where the $d_{220}$ interlattice spacing of magnetite was verified.

The magnetic properties were recorded with a (Quantum Design MPMS) SQUID magnetometer with field range of \pm 5 Tesla at the temperature range of 5-300 $\mathrm{K}$ and included hysteresis loops and ZFC-FC (zero field cooled - field cooled) cycles.

The heating efficiency of the nanoparticle solutions was measured by subjecting the solution to an $\mathrm{AC}$ magnetic field at $\mathrm{f}=210 \mathrm{kHz}(\mathrm{LF})$ and $765 \mathrm{kHz}(\mathrm{HF})$ at different concentrations varying from 0.3 to $1.2 \mathrm{mg}_{\mathrm{Fe}} / \mathrm{mL}$ and with field amplitudes $\mathrm{H}$ of 20 and $28 \mathrm{kAm}^{-1}$. A volume of $1 \mathrm{ml}$ from each solution was placed in the center of a water cooled induction coil of $23 \mathrm{~mm}$ diameter connected to an $\mathrm{AC}$ field generator. In each measurement, heating and cooling sequences were recorded for ample time ( $\leq 600 \mathrm{~s}$ for each sequence). Although the value $\mathrm{H} \times \mathrm{f}\left(\sim 15-21 \times 10^{9} \mathrm{Am}^{-1} \mathrm{~s}^{-1}\right)$ is set at least one order of magnitude above the estimated threshold for biological (human body) discomfort $\left(\sim 5 \times 10^{8} \mathrm{Am}^{-1} \mathrm{~s}^{-1}\right)$ [6] based on voluminous implants, analogous protocols have already been proposed in specific cases of smaller diameter body regions by direct injection with much shorter treatment times due to the enhanced MNPs heating efficiency.[7, 8] The hyperthermia efficiency is evaluated by measuring the specific loss power (SLP), which refers to the amount of energy converted into heat $(\mathrm{W})$ per time (Dt) and mass of the magnetic material $\left(\mathrm{Fe}_{3} \mathrm{O}_{4}\right.$ in this case). [9]

\section{Results and Discussion}

\subsection{Structure and Morphology}

In Fig.1 a TEM overview is shown. A lower magnification image is depicted in Fig.1a where random agglomerates of MNPs of 100-200 nm are in agreement with the hydrodynamic diameters listed in Table 1 . These agglomerates consist of MNPs of $12.3 \mathrm{~nm} \pm 20 \%$ as shown in the corresponding inset where the size-analysis and its log-normal distribution are given. The electron diffraction pattern (Fig.1b) verifies the existence of magnetite $\left(\mathrm{Fe}_{3} \mathrm{O}_{4}\right)$. The co-existence of maghemite, however, cannot be safely excluded since both phases possess major reflections within the experimental resolution. Fig.1c is a higher magnification image showing the typical overlapping of MNPs occurring within an aggregate area. Fig.1d is a HRTEM image reconstruction showing the highly crystalline nature of magnetite entities.

\subsection{Collective Magnetic behavior}

ZFC-FC magnetization data (Fig.2a) were recorded at 4 $\mathrm{kA} / \mathrm{m}$. It is evidenced that the system is in a superparamagnetic state at room temperature and remains so down to $250 \mathrm{~K}$ where the ZFC and FC branches separate. Hysteresis loops at low $(5 \mathrm{~K})$ and high temperature $(300 \mathrm{~K})$ yield a distinct saturation magnetization $\mathrm{Ms}(5 \mathrm{~K})=50$ and $\mathrm{Ms}(300 \mathrm{~K})=40 \mathrm{Am}^{2} / \mathrm{kg}$. The reduced magnetization with respect to bulk magnetite $\left(\sim 90 \mathrm{Am}^{2} / \mathrm{kg}\right)$ may be either attributed to the reduced size of constituent MNPs or the surface oxidation leading to formation of an antiferromagnetic $\mathrm{FeO}_{\mathrm{x}}$ shell. Superparamagnetism seems to dominate at room temperature shown by the lack of coercivity in Fig2b. In the inset of Fig. $2 b$ where the central part detail of the loops is shown, the transition from the superparamagnetic 
to ferromagnetic behavior is evident, since the low temperature loop exhibits a small yet non-zero coercivity.
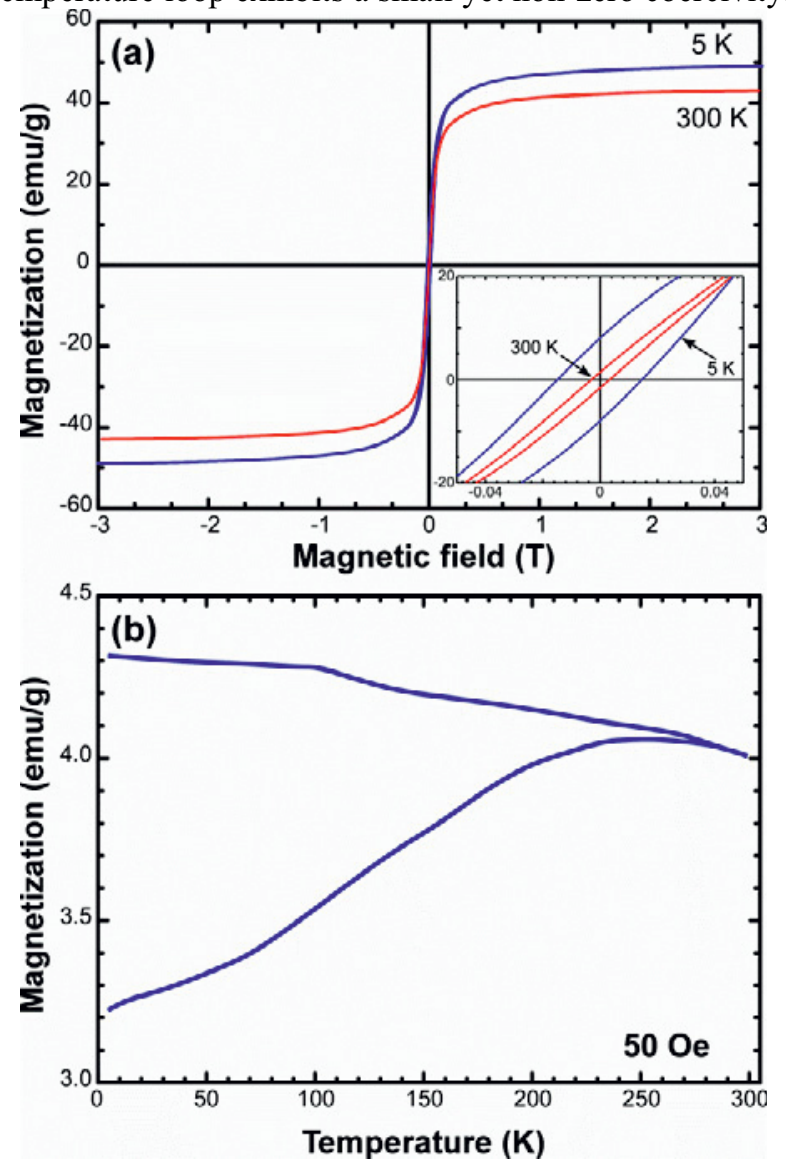

Fig. 2. SQUID magnetometry of dried nanoparticles (a) ZFCFC curves under $4 \mathrm{kA} / \mathrm{m}$, (b) Hysteresis loops recorded at 5 and $300 \mathrm{~K}$.

\subsection{Magnetic Particle Hyperthermia}

Fig.3a and Fig.3b show the experimental heating and cooling sequences for varying concentrations at $20 \mathrm{kA} / \mathrm{m}$ and LF $(210 \mathrm{kHz})$ and $\mathrm{HF}(765 \mathrm{kHz})$, respectively. In all cases an identical sequence is initially recorded for a pure aqueous (solvent) solution and used as reference to exclude heating exchange with the environment and eventually quantify accurately magnetic heating response via estimation of Specific Loss Power (SLP) values.[9] The shaded areas denote the desirable hyperthermia levels $\left(41-45{ }^{\circ} \mathrm{C}\right)$ required for a successful hyperthermal cell-shock. It seems that the LF requires a higher concentration of MNPs in order to provide a reliable result. On the other hand, the higher the frequency, the bigger the effect and as shown in Fig $3 \mathrm{~b}$ in the HF case even for the smaller solution concentration of 0.3 $\mathrm{mg}_{\mathrm{Fe}} / \mathrm{mL}$. The absence of hysteresis at $300 \mathrm{~K}$ facilitates the role of the available hyperthermia $\mathrm{AC}$ fields in transferring the maximum heating power to the dispersion, though as discussed later on anisotropy field should also be considered.

Hyperthermia efficiency is tuned by features that are highly dependent on particle size and also on the degree of aggregation of the particles, as we have reported previously. [10-13] Several heating mechanisms are possible: susceptibility loss, hysteresis loss and viscous heating as has been discussed in recent theoretical works to unravel the effect of frequency and optimum concentration towards maximum hyperthermia output.[14, 15] In this context, large variations of SLP with frequency and field intensity occur depending on the regime of the nanoparticles, which is governed by the relative magnitude of their anisotropy field $\left(\mathrm{H}_{\mathrm{A}}=2\right.$ $\mathrm{K} / \mathrm{M}_{\mathrm{S}}=40 \mathrm{kA} / \mathrm{m}$ for an assembly of uniaxial $\mathrm{Fe}_{3} \mathrm{O}_{4} \mathrm{MNPs}$ [14]) compared to that of the AC magnetic field (20, 28 $\mathrm{kA} / \mathrm{m}$ in our case).

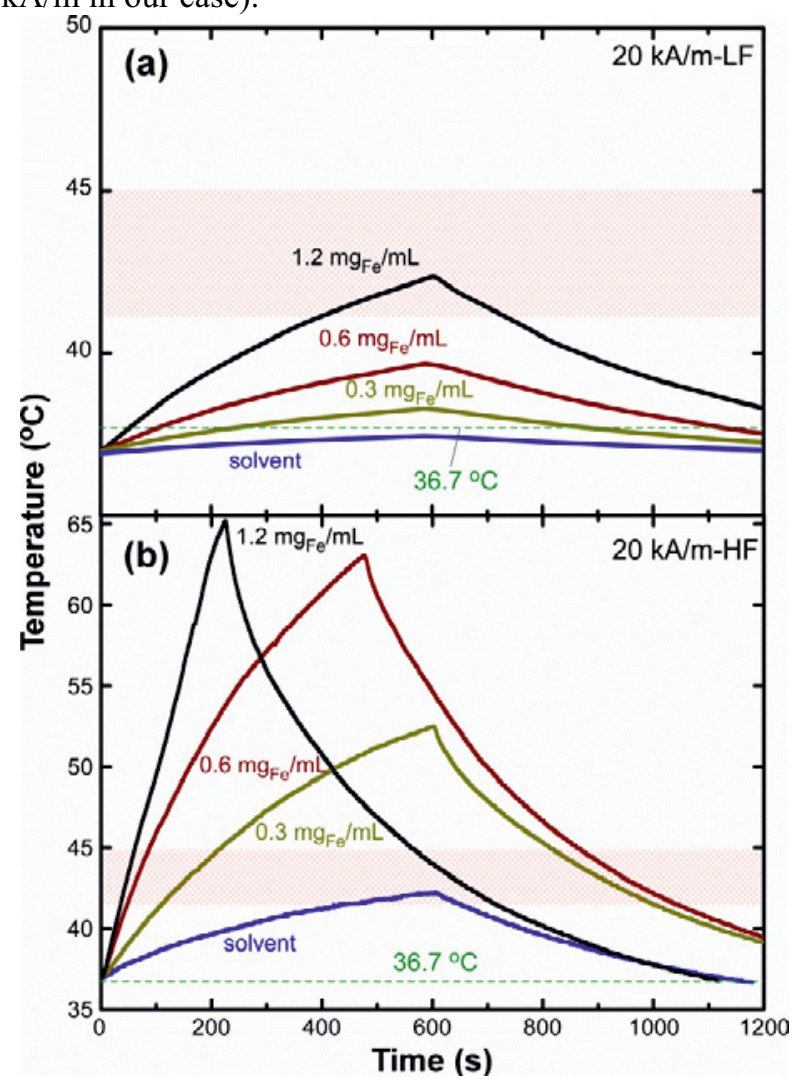

Fig. 3. Magnetic Hyperthermia heating-cooling cycles of fluidMAG-Streptavidin aqueous dilutions $(0.3, \quad 0.6,1.2$ $\mathrm{mg}_{\mathrm{Fe}} / \mathrm{mL}$ ) under low frequency (LF) i.e. $210 \mathrm{kHz}$ (a) and high frequency (HF) of $765 \mathrm{kHz}$ (b). Shaded bands in both graphs denote desirable hyperthermia levels.

In our case, $\mathrm{Fe}_{3} \mathrm{O}_{4}$ nanoparticles are well suited as hyperthermia agents in the intermediate region where $\mathrm{H}_{\mathrm{AC}} \sim 0.5 \mathrm{H}_{\mathrm{A}}$, according to the viscous, magnetodynamic and intermediate transition regions described by Usov and Liubimov in [15]. In Fig.4, the temperature records and the corresponding SLP values for all fields, frequencies and concentrations are compared. At lower frequencies $(210 \mathrm{kHz})$, the MNPs rotate in the liquid and the heating is mainly caused by viscous losses, so they exhibit attenuated SLP values, but to a different extent because of concentration variations. On the contrary, at higher frequencies $(765 \mathrm{kHz})$, MNPs are thermally driven to magnetodynamic region, leading to enhanced SLP values tunable with frequency and field intensity.

In an effort to put our SLP findings into a broader context we compared our results with the data in the review of Kallumadil et al.[2]. Among them, the Chemicell products of similar type (Fluidmag-D Starch: 
$50 \mathrm{~nm}$ 'fresh', $50 \mathrm{~nm}$ 'aged' (9 months), $100 \mathrm{~nm}$ ) measured at $5 \mathrm{mg} / \mathrm{mL}$ (Fe concentration), $900 \mathrm{kHz}, 5.66$ $\mathrm{kA} / \mathrm{m}$ possess SLP values 38, 77 and $58 \mathrm{~W} / \mathrm{g}$, respectively.

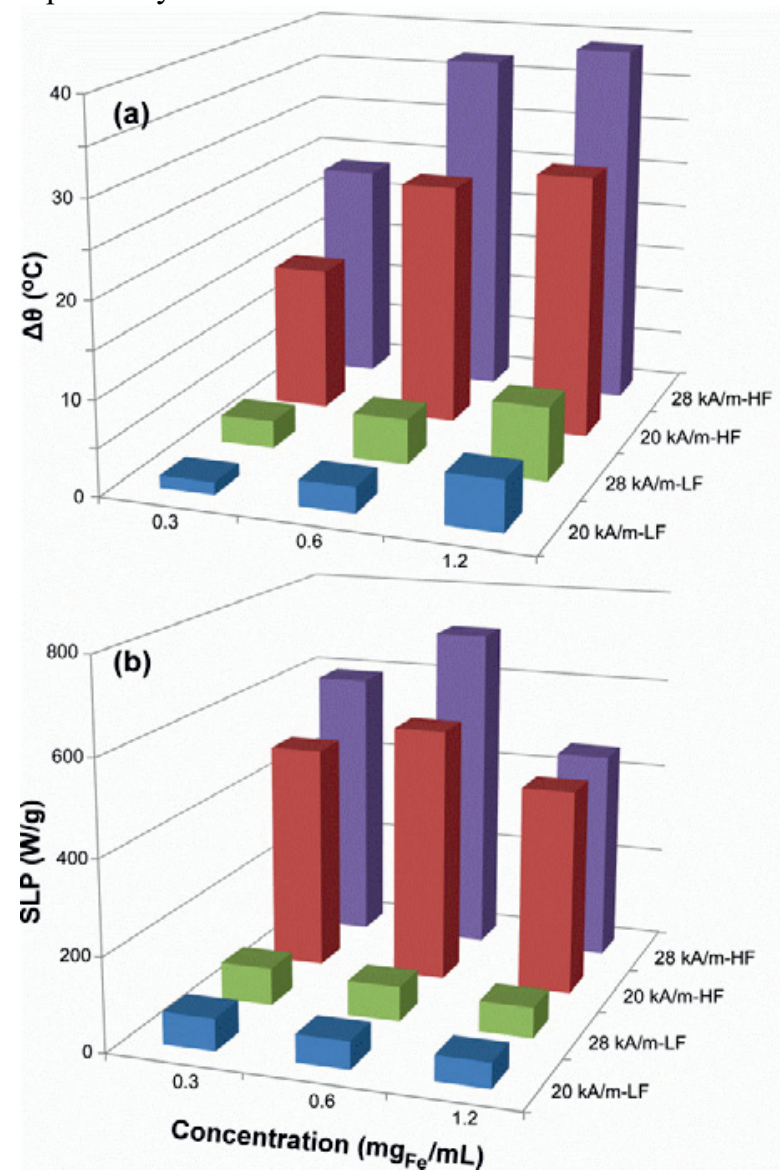

Fig. 4. Magnetic hyperthermia quantification measures of fluidMAG-Streptavidin aqueous dilutions $(0.3,0.6,1.2$ $\mathrm{mg}_{\mathrm{Fe}} \mathrm{mL}$ ) under low frequency (LF) i.e. $210 \mathrm{kHz}$ and high frequency (HF) of $765 \mathrm{kHz}$ and variable magnetic field intensity $(20,28 \mathrm{kA} / \mathrm{m})$ expressed as temperature variations (in $\left.{ }^{\circ} \mathrm{C}\right)(\mathrm{a})$ and Specific Loss Power Ratio (in W/g) (b).

As discussed by Kallumadil et al., Chemicell's 'aged' fluidmag-D50nm showed significantly more heating efficiency than a nominally identical, but freshly prepared batch of the same sample. The difference (from 38 to 77 $\mathrm{W} / \mathrm{g}$ ) was attributed to some aging of the fluid, as evidenced by a larger crystallite size being found for the older sample. Our much higher efficiencies (up to 710 $\mathrm{W} / \mathrm{g}$ at $765 \mathrm{kHz}, 28 \mathrm{kA} / \mathrm{m}, 0.6 \mathrm{mgFe} / \mathrm{mL}$ ) may be attributed to the smaller concentration solution that allow for better manipulation of MNPs inside the AC magnetic field and also to higher intensity magnetic fields overcoming the constraints of coercivity and anisotropy field of the MNPs.

Additionally, as Fig.5 shows, MNPs tend to aggregate in bigger assemblies as they age. This effect is mainly governed by dipolar interactions and as we discussed in [12] chain formation significantly enhances hyperthermia response, since it drastically affects hysteresis loop features. These large-scale arbitrary, yet longitudinal formations of MNPs may be further controlled, if colloidal stability is properly adjusted via solution viscosity. Chains of specific orientations may be selectively guided to form and freeze and lead easily to even more enhanced hyperthermia efficiency.

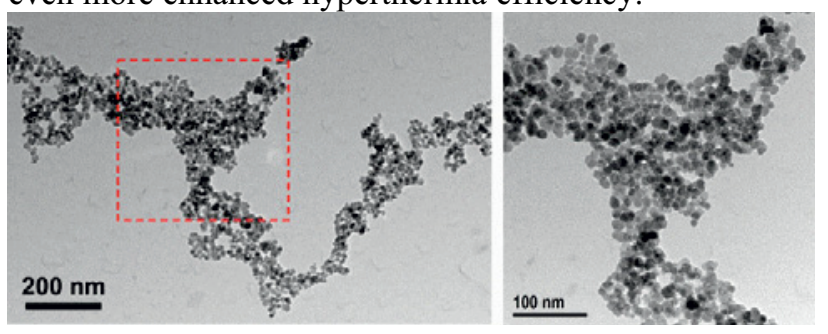

Fig. 5. Aging Effect: TEM imaging (left image) and detail of central part (right image) indicating the large-scale oriented (chain-like) assembly formations resulting to enhanced hyperthermia performance.

\section{Conclusions}

"FluidMAG" (from Chemicell GmbH), a commercial ferrofluid actually employed for purification or separation of biomolecules, was examined as a magnetic hyperthermia agent. Its structural and magnetic features were found in good agreement with company datasheet and correlated with its heating response. This product shows a significant heating response that can be tuned by field and frequency of the AC driving field and the solution concentration.

Financial support by DAAD-IKYDA Greek-German Bilateral Cooperation Program is acknowledged.

\section{References}

1. C. S. S. R Kumar, F. Mohammad Advanced Drug Delivery Reviews 63, 789-808 (2011).

2. M. Kallumadil, M. Tada, T.Nakagawa, M. P. Southern, Q. A.Pankhurst, J. Magn. Magn. Mater. 321, 1509 (2009).

3. J.Gao, H. Gu and B. Xu, Acc.Chem.Res. 42 [8], 1097 (2009).

4. J. Xie, G. Liu, H. S. Eden, H. Ai, X. Chen Accounts of Chemical Research Vol. 44, No. 10 883-892 (2011).

5. http://www.chemicell.com/products/ferrofluid/ferrofluids.html

6. R. Hergt, S. Dutz, R. Müller and M. Zeisberger, J. Phys.: Condens. Matter 18, S2919 (2006).

7. Q.A. Pankhurst, N.K.T. Thanh, S.K. Jones and J. Dobson, J. Phys. D: Appl. Phys, 42, 224001 (2009).

8. S.E. Barry, Int. J. Hyperthermia 24, 451-466 (2008).

9. A. Chalkidou, K. Simeonidis, M. Angelakeris, T. Samaras, C. Martinez-Boubeta, Ll. Balcells, K. Papazisis, C. DendrinouSamara, O. Kalogirou, J. Magn. Magn. Mater. 323, 775 (2011).

10. K. D. Bakoglidis, K. Simeonidis, D. Sakellari, G. Stefanou and M. Angelakeris, IEEE Trans. Magn. 48, 1320 (2012).

11. D. Serantes, D. Baldomir, C. Martinez-Boubeta, K. Simeonidis, M. Angelakeris, E. Natividad, M. Castro, D.-X. Chen, A. Sanchez, Ll. Balcells and B. Martínez: J. Appl. Phys. 108, 073918 (2010).

12 C. Martinez-Boubeta, K. Simeonidis, David Serantes, I. CondeLeborán, I. Kazakis, G. Stefanou, L. Peňa, R. Galceran, Ll. Balcells, C. Monty, D. Baldomir, M. Mitrakas, and M. Angelakeris, Adv. Func. Mater. 22, 3737-3744 (2012).

13 C. Martinez-Boubeta, K. Simeonidis, A. Makridis, M. Angelakeris, O. Iglesias, P. Guardia, A. Cabot, Ll. Yedra, S. Estradé, F. Peiró, Z. Saghi, P. A. Midgley, I. Conde-Leborán, D. Serantes, D. Baldomir, Scientific Reports, 3:1652 (2013).

14 J. Carrey, B. Mehdaoui, and M. Respaud, J. Appl. Phys. 109, 083921 (2011).

15 N. A. Usov and B. Ya. Liubimov, J. Appl. Phys. 112, 023901 (2012). 